\title{
Clustering of Cochlear Oscillations in Frequency Plateaus as a Tool to Investigate SOAE Generation
}

Epp, Bastian; Wit, Hero ; van Dijk, Pim

Published in:

AIP Conference Proceedings

Link to article, DOI:

$10.1063 / 1.4939423$

Publication date:

2016

Document Version

Publisher's PDF, also known as Version of record

Link back to DTU Orbit

Citation (APA):

Epp, B., Wit, H., \& van Dijk, P. (2016). Clustering of Cochlear Oscillations in Frequency Plateaus as a Tool to Investigate SOAE Generation. AIP Conference Proceedings, 1703, [090025]. https://doi.org/10.1063/1.4939423

\section{General rights}

Copyright and moral rights for the publications made accessible in the public portal are retained by the authors and/or other copyright owners and it is a condition of accessing publications that users recognise and abide by the legal requirements associated with these rights.

- Users may download and print one copy of any publication from the public portal for the purpose of private study or research.

- You may not further distribute the material or use it for any profit-making activity or commercial gain

- You may freely distribute the URL identifying the publication in the public portal 


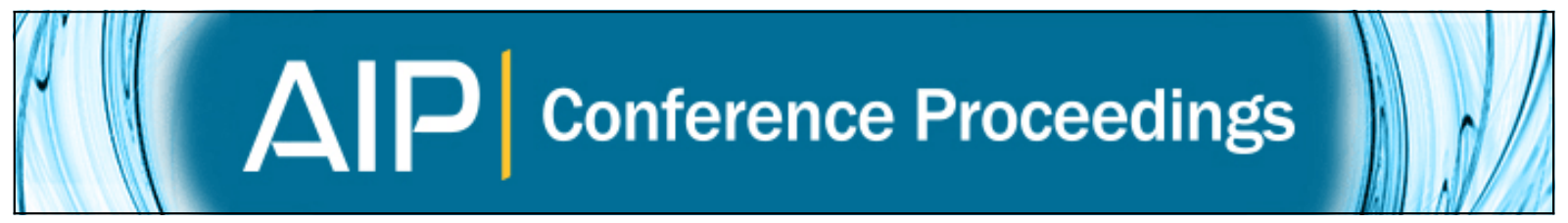

Clustering of cochlear oscillations in frequency plateaus as a tool to investigate SOAE generation

Bastian Epp, Hero Wit, and Pim van Dijk

Citation: AIP Conference Proceedings 1703, 090025 (2015); doi: 10.1063/1.4939423

View online: http://dx.doi.org/10.1063/1.4939423

View Table of Contents: http://scitation.aip.org/content/aip/proceeding/aipcp/1703?ver=pdfcov

Published by the AIP Publishing

\section{Articles you may be interested in}

Flare-generated coronal loop oscillations: A tool for MHD coronal seismology

AIP Conf. Proc. 537, 264 (2000); 10.1063/1.1324949

Speech generation using variable frequency oscillators

J. Acoust. Soc. Am. 95, 1184 (1994); 10.1121/1.408457

Are spontaneous otoacoustic emissions generated by self-sustained cochlear oscillators?

J. Acoust. Soc. Am. 89, 2391 (1991); 10.1121/1.400958

Frequency-domain investigations of cochlear stability in the presence of active elements

J. Acoust. Soc. Am. 73, 1244 (1983); 10.1121/1.389272

Frequency-domain investigations of cochlear stability in the presence of negative damping

J. Acoust. Soc. Am. 71, S17 (1982); 10.1121/1.2019251 


\title{
Clustering of Cochlear Oscillations in Frequency Plateaus as a Tool to Investigate SOAE Generation
}

\author{
Bastian Epp*, Hero Wit ${ }^{\dagger}$ and Pim van Dijk ${ }^{\dagger}$ \\ ${ }^{*}$ Hearing Systems - Oticon Centre of Excellence for Hearing and Speech Sciences (CHeSS), Department of \\ Electrical Engineering, Technical University of Denmark, Lyngby, Denmark \\ $\dagger$ Department of Otorhinolaryngology, University Medical Center Groningen, University of Groningen,
} Groningen, The Netherlands

\begin{abstract}
Spontonaeous otoacoustic emissions (SOAE) reflect the net effect of self-sustained activity in the cochlea, but do not directly provide information about the underlying mechanism and place of origin within the cochlea. The present study investigates if frequency plateaus as found in a linear array of coupled oscillators (OAM) [7] are also found in a transmission line model (TLM) which is able to generate realistic SOAEs [2] and if these frequency plateaus can be used to explain the formation of SOAEs. The simulations showed a clustering of oscillators along the simulated basilar membrane Both, the OAM and the TLM show traveling-wave like behavior along the oscillators coupled into one frequency plateau. While in the TLM roughness is required in order to produce SOAEs, no roughness is required to trigger frequency plateaus in the linear array of oscillators. The formation of frequency plateaus as a consequence of coupling between neighbored active oscillators might be the mechanism underlying SOAEs.
\end{abstract}

\section{INTRODUCTION}

Spontaneous otoacoustic emissions (SOAE) are time-pressure signals that can be measured in the ear canal of numerous species in the absence of external stimulation. It is commonly assumed that the active process linked to hair cell motility is the main element contributing to SOAE generation. The exact generation mechanism is however still under debate. SOAEs have a preferred frequency spacing and a distribution of energy around their center frequency. The preferred frequency spacing is in line with the hypothesis of coherent reflection of traveling waves within the cochlea between local impedance mismatches and the oval window (linear reflection theory, [4]). Another interpretation of the preferred frequency spacing is the grouping of neighboring segments of the cochlea into groups of oscillators (frequency plateaus) with a common frequency by nonlinear entrainment effects. The distribution of energy around their center frequency are in line with the model assumption that the cochlea can be modeled as a chain of coupled, nonlinear and active oscillators including entrainment effects. SOAEs of humans have been modeled along with other data from cochlear processing using one-dimensional transmission lines (TLM) with fluid coupling $[2,3,5]$. These transmission line models are also capable of accounting for entrainment effects of SOAEs to puretone stimulation [1]. Frequency plateaus hypothesized to underly SOAES of lizards have successfully been modeled by a chain of nonlinear oscillators (linear array model, OAM) with nearest-neighbor coupling and varied mechanical properties [7]. It was recently shown that the parameters in such a linear array can be modified such that frequency plateaus arising from nonlinear interaction of the coupled oscillators have a preferred frequency separation in line with data from human SOAEs. This finding indicates a common physical mechanism underlying SOAE generation in the human cochlea and the basilar papilla of lizards. Besides the similarity of the two models, some open questions remain. While impedance mismatches introduced by stochastic variation of local mechanical parameters are essential to generate SOAEs in the TLM, frequency plateaus generated in the OAM as used in [7] are hardly affected by introduction of impedance mismatches. Another important element of the linear reflection theory is the presence of backward traveling waves. While these backward traveling waves are feasible in the TLM due to the boundary conditions at the oval window and the helicotrema, the OAM does not explicitly account for these boundary effects. A more detailed understanding of the common underlying mechanisms between mammalian and lizard SOAE generation will help to strengthen the transfer of knowledge obtained in animal models towards human hearing. 


\section{MODELS}

\section{Linear Array Model (OAM)}

The formation of frequency plateaus was investigated using a OAM with 241 segments written in the normal form of a Hopf bifurcation ([6]; all parameters taken from [7]):

$$
\begin{array}{rr}
\dot{z}_{j}=\left(i \omega_{j}+\varepsilon_{j}\right) z_{j}+\kappa_{j}\left(d_{R}+i d_{I}\right)\left(z_{j-1}-2 z_{j}+z_{j+1}\right)-B_{j}\left|z_{j}\right|^{2} z_{j} \\
x_{j} & \text { displacement of j-th oscillator } \\
z_{j} & x_{j}-\frac{1}{\omega_{j}} i \dot{x}_{j} \\
\varepsilon_{j} & \text { effective damping } \\
\kappa_{j} & \text { relative coupling strength } \\
d_{R} & \text { dissipative coupling } \\
d_{I} & \text { reactive coupling } \\
B_{j} & \text { nonlinearity factor }
\end{array}
$$

The model equations were solved in the time domain using MATHEMATICA (procedure 'NDSolve', method option 'Automatic') for a $500 \mathrm{~ms}$ long time interval. Roughness was introduced into the model by stochastic variation of the place-frequency map by replacing $\omega_{j} \rightarrow \omega_{j}+\Delta \omega$ with $\Delta \omega$ taken from a normal distribution with mean 0 and standard deviation of $1 \%$ of the median of all $\omega_{j}$ ).

\section{One-Dimensional Transmission Line Model (TLM)}

A nonlinear and active TLM with 1000 segments and included roughness of the cochlea was used to simulate SOAEs (all parameters taken from [2]):

$$
\begin{array}{lr}
p_{j}=m \ddot{x}_{j}+d_{j}\left(\dot{x}_{j}\right) \dot{x}_{j}+s_{j}\left[x_{j}+\left.c_{j}\left(\dot{x}_{j}\right) \dot{x}_{j}(t)\right|_{t-\tau}\right] \\
p_{j} & \text { pressure at } \mathrm{j} \text {-th oscillator } \\
m & \text { effective mass of } \mathrm{j} \text {-th oscillator } \\
x_{j} & \text { displacement of } \mathrm{j} \text {-th oscillator } \\
d_{j}\left(\dot{x}_{j}\right) & \text { nonlinear damping coefficient } \\
s_{j} & \text { linear spring constant of } \mathrm{j} \text {-th element } \\
c_{j}\left(x_{j}\right) & \text { nonlinear feedback stiffness term } \\
\tau & \text { feedback time delay }
\end{array}
$$

The model equations were solved in the time domain with a rate of $400 \mathrm{kHz}$ using a modified 4-th order Runge-Kutta method.

\section{SIMULATIONS}

In the OAM, frequency plateaus were simulated with parameters that lead to a preferred frequency separation of the plateaus in line with human data [7]. In the TLM, SOAEs were simulated with the same parameters used in [2]. For comparison of the OAM and TLM simulations, the temporal dynamics of the simulated cochlea was analyzed and compared to the temporal dynamics of the OAM. A combination of dissipative and reactive coupling was included in the OAM to compare the simulations with the results of [6] and [7]. To facilitate the comparison of the OAM with the TLM (where coupling between segments is modeled by a reactive element), in a second set of simulations only reactive coupling and included roughness was used in the OAM.

\section{Linear Array Model (OAM)}

The segments in the OAM were adjusted to match the place-frequency map and the spatial segment separation as in [2]. The 241 segments of the OAM were mapped into the frequency range of 0.88 to $3.13 \mathrm{kHz}$, spanning the central part of the cochlea. The initial values for the simulation were $z_{j}(0)=0.01$. The parameters of the model were $\varepsilon_{j}=1, B_{j}=1, d_{R}=0.2$ and $d_{I}=-1.5$. To reduce boundary effects, the values of $\kappa_{j}$ were adjusted to $k_{j}=0.1 \cdot j$ for $j=\{1,2, \ldots, 9\}$ and to $\kappa_{j}=1-0.1 \cdot j$ for $j=\{n-9, n-8, \ldots, n\}$ and 1 for all other $j$. The last $50 \mathrm{~ms}$ of the simulated $x_{j}=\mathfrak{R}\left\{z_{j}(t)\right\}$ was windowed using a Hann window, zero-padded to a length of $250 \mathrm{~ms}$ and the absolute value of the FFT calculated. 

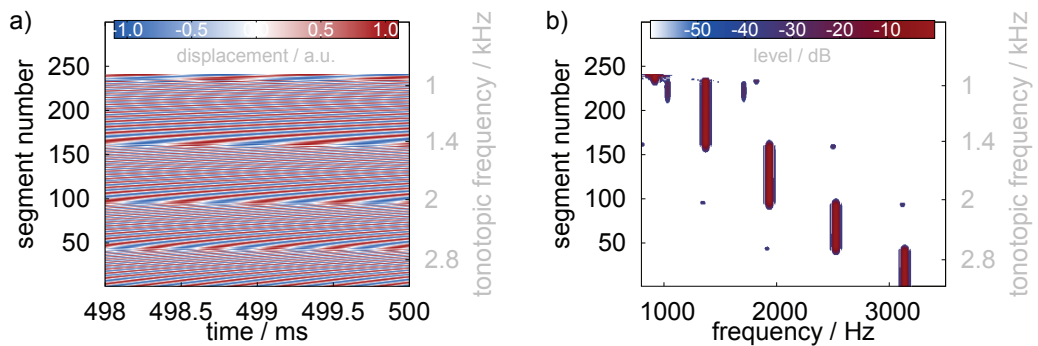

FIGURE 1. Temporal dynamics (a, time interval from 498-500 ms) and frequency analysis (b) of the OAM in a steady-state. The right ordinate shows the tonotopic frequency of the corresponding segment number on the left ordinate.
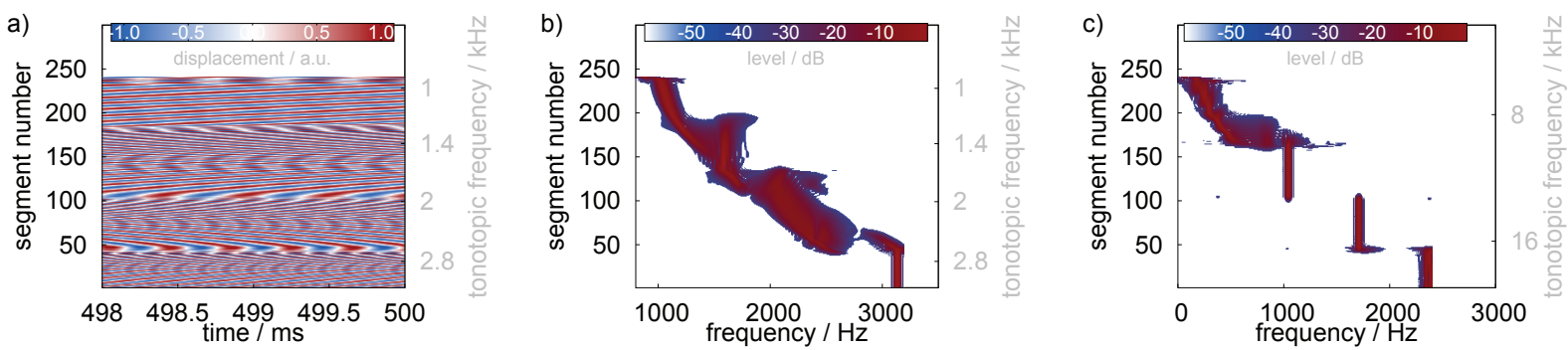

FIGURE 2. Same as Fig. 1, but for reactive coupling only $\left(d_{R}=0\right)$ without roughness (b) and with included roughness (c).

\section{Self-Sustained Activity and Frequency Plateaus}

The steady-state displacements of the OAM including capacitive and reactive coupling is shown in Fig. 1a. The frequency analysis of the temporal dynamics of each segment is shown in Fig. 1b. The temporal dynamics of the OAM show a broad activity over all simulated segments with some visible boundaries around segments 50, 100, and 180. Frequency analysis of the temporal dynamics show a clustering of the segments around specific frequencies. The segments clustering around one frequency are all connected to their direct neighbors, exposing closed frequency plateaus along the tonotopic organization of the modeled array.

The steady-state displacements of the model including reactive coupling only is shown in Fig. 2a. The frequency analysis of the temporal dynamics of each segment is shown in Fig. 2b without roughness and in Fig. 2c with included roughness. The temporal dynamics of the OAM show a broad activity over all simulated segments. Without inclusion of roughness of the parameters, the clustering of the segments is less salient (Fig. 2b). Only at high frequencies, some clustering remains. The energy of other segments is distributed over a rather broad spectral range. With inclusion of roughness, neighboring segments show a clear tendency to cluster into plateaus (Fig. 2c).

\section{Transmission Line Model (TLM)}

SOAEs were simulated for a duration of $1 \mathrm{~s}$. The initial conditions for the SOAE simulations were set to the steady state of the model after transient stimulation with a click and 1s subsequent simulation time. SOAEs were extracted computed using a FFT of the ear canal signal after windowing with a Hann window [2]. To obtain information about the dynamics of the single segments, the velocity of the segments over time was frequency analyzed. To evaluate the formation of plateaus, the frequency with maximum energy was extracted and used as the dominant frequency for the corresponding segment. To investigate the presence of multiple reflections and hence standing waves between peak region and the oval window, the temporal dynamics of the TLM was band-pass filtered around the frequency of one SOAE (or, equivalently one frequency plateau).

\section{Self-Sustained Activity, Spontaneous Otoacoustic Emissions and Frequency Plateaus}

The self-sustained oscillations of the TLM are shown for each segment in Fig. 3a. The frequency analysis of the temporal dynamics is shown in Fig. 3b. The temporal dynamics of the segments (Fig. 3a) shows high activity in the 

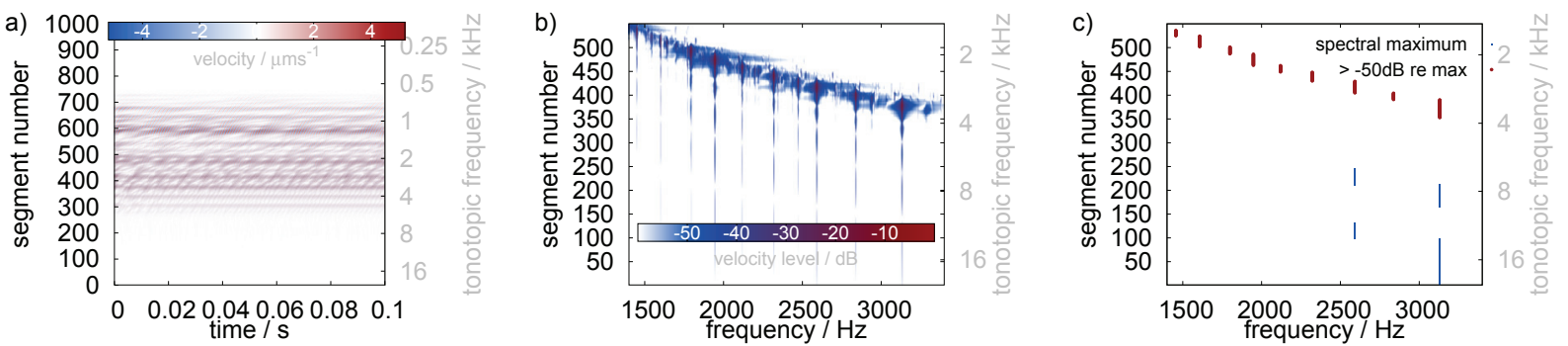

FIGURE 3. Same as Fig. 1, but for the TLM and with extracted frequency plateaus (c).
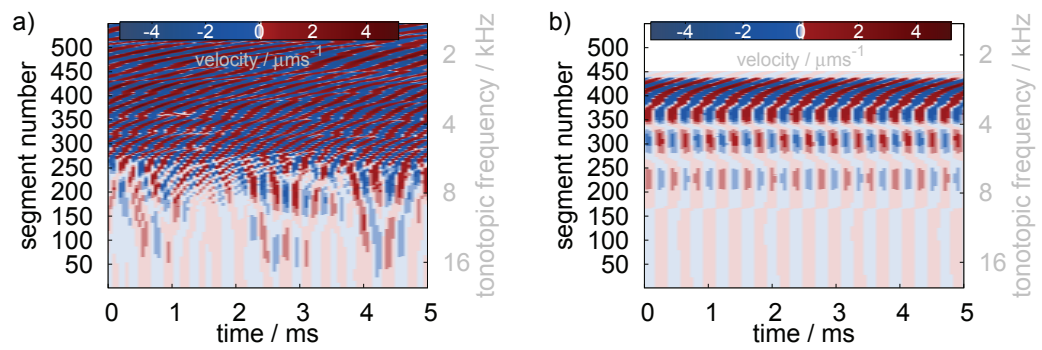

FIGURE 4. Temporal dynamics of the TLM in the basal region of the simulated cochlea including all frequencies (a) and after band-pass filtering (b). The dynamics of the TLM was band-pass filtered with a 4th order Butterworth filter centred at $2600 \mathrm{~Hz}$ (around the frequency of a SOAE) and a bandwidth of $100 \mathrm{~Hz}$.

mid-region of the modeled cochlea corresponding to tonotopic frequencies between $700 \mathrm{~Hz}$ and $4000 \mathrm{~Hz}$, in line with the frequency range of the simulated SOAEs in the ear canal (see [2]). The spectral analysis of the temporal dynamics (Fig. 3b) shows the highest energy density around the frequencies corresponding to the simulated SOAEs and the places corresponding to the tonotopic frequency of segments. The analysis also reveals some dominant frequency regions with energy in a narrow spectral range. At these dominant frequencies, energy can be found over a broad range of segments (visible as vertical stripes). This might indicate the presence of frequency plateaus, i.e., the dominance of one frequency within a group of adjacent oscillators. Another observation is the presence of small gaps within these groups of oscillators, i.e., spatially localized minimum of oscillatory energy at this frequency. Frequency plateaus extracted from the data in Fig. 3b are shown in Fig. 3c. Frequency plateaus could be found along the whole length of the modeled cochlea. The frequency plateaus are roughly arranged along the tonotopic frequency map. Some frequencies show multiple, spatially separated frequency plateaus. The frequency plateaus have varied spatial extent and tend to be closer and more narrow at low frequencies. A closer analysis of the amplitudes within the frequency plateaus (Fig. 3c) shows a difference in amplitude for different plateaus at the same frequency of $50 \mathrm{~dB}$ or more. A standing-wave like pattern as predicted by the theory of multiple reflections might not be visible in the peak region of the traveling wave since the relative amplitude of the forward-traveling might be too high compared to the reflected wave. Hence, a standing-wave like pattern would be expected in the basal region of the cochlea where the backward-traveling wave and the reflection back into the cochlea with forward-amplification have more similar amplitudes. Fig. 4a shows temporal dynamics of the TLM from Fig. 3 for the basal segments, and the same segments, but band-pass filtered around the frequency of a $2600 \mathrm{~Hz}$ (Fig. 4b). While in Fig. 4a, mainly forward-traveling waves are present, the band-pass filtered segments reveal the presence of nodes and antinodes and hence a standing-wave pattern at the basal region. Close to the tonotpic place of the filtered component (around segment 400), a forward-traveling wave can be observed.

\section{DISCUSSION}

Both, OAM and TLM produce frequency plateaus. The OAM produces frequency plateaus without inclusion of roughness if the coupling is dissipative and reactive, but roughness is required if only reactive coupling is included. This is consistent with the fact that in the TLM, segments are coupled via a reactive element (the fluid) and roughness is required to generate SOAEss. In the TLM, the frequencies of the plateaus correspond to the frequencies of the 
SOAEs in the ear canal. This shows that a group of entrained oscillators underlies the generation of SOAEs.

The temporal dynamics of both models show a clear inward traveling wave pattern from high to low tonotopic frequencies, suggesting the absence of standing waves as postulated in the linear reflection theory. In the basal region of the TLM however, a clear standing wave pattern could be found. Hence, an interaction between forward- and backward traveling wave may also be present in the region of maximum amplitude which might however not be observable due to the large amplitude of the forward traveling wave.

The inclusion of roughness for purely reactive coupling causes reflection of the forward traveling wave. The necessity of roughness to generate frequency plateaus indicates that the reflection near the peak region plays a major role in the generation of the plateaus. The generation of backward traveling waves via linear reflection and the interaction of forward- and backward traveling waves seem to be the critical elements determining the frequencies of the plateaus and consequently of the SOAEs found in the ear canal, supporting the linear reflection theory by [4]. In a TLM model that incorporates reactive and dissipative coupling between the elements, the roughness is probably not necessary to produce frequency plateaus. The reactive coupling is based on fluid coupling between sections in the model. In contrast, the dissipative coupling does not have a known physiological correlate in the cochlea, and remains speculative.

\section{ACKNOWLEDGMENTS}

This work was supported by the Oticon Centre of Excellence for Hearing and Speech Sciences at the Technical University of Denmark and the Heinsius Houbolt Foundation.

\section{REFERENCES}

[1] Epp B, Mauermann M, Verhey JL (2011) Cochlear fine structure - implications for modulation processing at the level of the cochlea. In: Shera CA, Olson ES (eds) What Fire is in Mine Ears: Progress in Auditory Biomechanics, Melville: American Institute of Physics, pp. 206-211

[2] Epp B, Verhey JL, Mauermann M (2010) Modeling cochlear dynamics: interrelation between cochlea mechanics and psychoacoustics. J Acoust Soc Am 128:1870-1883

[3] Mauermann M, Uppenkamp S, van Hengel PW, Kollmeier B (1999) Evidence for the distortion product frequency place as a source of distortion product otoacoustic emission (DPOAE) fine structure in humans. I. Fine structure and higher-order DPOAE as a function of the frequency ratio f2/f1. J Acoust Soc Am 106:3473-3483

[4] Shera CA (2003) Mammalian spontaneous otoacoustic emissions are amplitude-stabilized cochlear standing waves. J Acoust Soc Am 114:244-262

[5] Talmadge CL, Tubis a, Long GR, Piskorski P (1998) Modeling otoacoustic emission and hearing threshold fine structures. J Acoust Soc Am 104:1517-43

[6] Vilfan A, Duke T (2008) Frequency clustering in spontaneous otoacoustic emissions from a lizard's ear. Biophys J 95:46224630

[7] Wit HP, van Dijk P (2012) Are human spontaneous otoacoustic emissions generated by a chain of coupled nonlinear oscillators? J Acoust Soc Am 132:918-926

\section{COMMENTS AND DISCUSSION}

Geoff Manley: The result that "The frequency plateaus have varied spatial extent and tend to be closer and more narrow at low frequencies" corresponds to the finding that in humans, lower-frequency SOAE peaks are much closer to each other $(\sim 75 \mathrm{~Hz}$ at 1 $\mathrm{kHz})$ than higher-frequency SOAE $(220 \mathrm{~Hz}$ at $4 \mathrm{kHz})$.

Andrew Bell: You show interesting clustering effects. Does the frequency ratio between the clusters depend on kappa (or on d-sub-I)?

Robert Withnell: How does the parameter for the effective damping, $\varepsilon$, relate to the distance of each Hopf oscillator from the bifurcation point? A coupled-oscillator model constrains the individual oscillators so that each individual oscillator will no longer reach the bifurcation point. How does this impact self-sustained oscillation? For what coupling strength is self-sustained oscillation suppressed? What is the expected strength of viscous coupling?

Chris Bergevin: One minor comment: I'd suggest getting rid of the nomenclature 'LAM' [linear array model]. The 'linear' aspect seems to me to be misleading in that you are ultimate referring to a collection on *nonlinear* oscillators by virtue of their equations of motion. The physics of the underlying oscillators should be the crucial distinction, not the geometry (I assume 'linear' simply means they are arranged in a line?; if they were in an arc, would it then instead be a 'NLAM'?). For what it is worth, I'm not sure 
the geometry is a crucial distinction here in these 1-D models. At least for the lizard, given papillar coupling (which was ignored by Vilfan \& Duke 2008; see Bergevin \& Shera 2010 Sec.V.E for a related discussion).

Bastian Epp [reply to comments above]: First of all thanks for the comments and "sorry" for the long delay.

[Reply to Andrew Bell]: For the distance of the plateaus: There is a longer list of thing we need to investigate systematically - I will have to put this in the stack and am not sure I will have an answer until next week...

[Reply to Robert Withnell]: These are excellent questions. As to this point, findings are preliminary on the sense that the phenomenon can be observed in both approaches. The next step is to bring the two models together in the same framework, but I have to admit I did not have a smart idea of formulating the time-delay term of the TLM into the normal form of a Hopf-bifurcation. We will check the Bifurcation diagrams for the more simple model and the (more or less empirically found) parameters and will see what the dynamics is ... I do not have ready-made scripts for that yet, so as for Andrew, I do not yet have an answer until next week but will have it after the workshop (I hope).

[Reply to Chris Bergevin]: Perfectly correct. Changed on the poster and will be changed in the MS.

Martin Braun: Comment on the distance of the "frequency plateaus"; also referring to Geoff Manley's reply above. In mammalian hearing the behavioral frequency selectivity is logarithmic. It is therefore better described in frequency ratios, rather than in absolute frequency values. The established unit for this purpose is the octave (ratio 2:1). In behavioral acoustics of humans, there is also an established subunit of the octave: the Cent unit ( 1 octave $=1200$ Cent $)$.

Comprehensive analyses of SOAE frequency distribution in human ears showed a preferred minimum distance (PMD) of ca 100 Cent (Braun 1997). This value varies little across the main frequency range of recorded SOAEs (1-4 kHz). But, interestingly, it varies significantly between ears with few emissions, showing a PMD of ca 90 Cent, and ears with many emissions, showing a PMD of ca 100 Cent (Fig. 4 of Braun 2013). How would your model be related to these data?

Brown M (2013) High-multiple spontaneous otoacoustic emissions confirm theory of local tuned oscillators. SpringerPlus 2:135

Bastian Epp [reply to Martin Braun]: Hi Martin! Thanks for raising this interesting questions. As for the current manuscript, we were mainly concerned about the underlying mechanism, but it is interesting to simulate a couple of cochleas and to look at the statistics (similar to the contribution of Fruth et al.). I will consider this and get back to you with the results. 